\title{
Non-invasive urinary metabolomic profiling discriminates prostate cancer from benign prostatic hyperplasia
}

\author{
Clara Pérez-Rambla ${ }^{1}$ - Leonor Puchades-Carrasco ${ }^{1}$ María García-Flores $^{2}$ • \\ José Rubio-Briones $^{3} \cdot$ José Antonio López-Guerrero $^{2} \cdot$ Antonio Pineda-Lucena $^{1,4}$
}

Received: 26 August 2016 / Accepted: 2 March 2017 / Published online: 9 March 2017

(c) The Author(s) 2017. This article is an open access publication

\begin{abstract}
Introduction Prostate cancer $(\mathrm{PCa})$ is one of the most common malignancies in men worldwide. Serum prostate specific antigen (PSA) level has been extensively used as a biomarker to detect PCa. However, PSA is not cancerspecific and various non-malignant conditions, including benign prostatic hyperplasia (BPH), can cause a rise in PSA blood levels, thus leading to many false positive results.

Objectives In this study, we evaluated the potential of urinary metabolomic profiling for discriminating PCa from $\mathrm{BPH}$. Methods Urine samples from $64 \mathrm{PCa}$ patients and 51 individuals diagnosed with BPH were analysed using ${ }^{1} \mathrm{H}$ nuclear magnetic resonance $\left({ }^{1} \mathrm{H}-\mathrm{NMR}\right)$. Comparative analysis of urinary metabolomic profiles was carried out using multivariate and univariate statistical approaches.
\end{abstract}

Results The urine metabolomic profile of $\mathrm{PCa}$ patients is characterised by increased concentrations of

The original version of this article was revised due to a retrospective Open Access order.

Clara Pérez-Rambla and Leonor Puchades-Carrasco are joint first authors with equal contribution.

Antonio Pineda-Lucena

pineda_ant@gva.es

1 Structural Biochemistry Laboratory, Centro de Investigación Príncipe Felipe, 46012 Valencia, Spain

2 Laboratory of Molecular Biology, Fundación Instituto Valenciano de Oncología, 46009 Valencia, Spain

3 Department of Urology, Fundación Instituto Valenciano de Oncología, 46009 Valencia, Spain

4 Drug Discovery Unit, Instituto de Investigación Sanitaria La Fe, Avda. Fernando Abril Martorell, 106, 46026 Valencia, Spain branched-chain amino acids (BCAA), glutamate and pseudouridine, and decreased concentrations of glycine, dimethylglycine, fumarate and 4-imidazole-acetate compared with individuals diagnosed with $\mathrm{BPH}$.

Conclusion PCa patients have a specific urinary metabolomic profile. The results of our study underscore the clinical potential of metabolomic profiling to uncover metabolic changes that could be useful to discriminate PCa from BPH in a clinical context.

Keywords Biomarkers - Metabolomics - Prostate cancer · Benign prostatic hyperplasia $\cdot$ Nuclear magnetic resonance
Abbreviations
$\mathrm{PCa}$
Prostate cancer
PSA
Prostate specific antigen
DRE
Digital rectal examination
${ }^{1} \mathrm{H}-\mathrm{NMR}$
Nuclear magnetic resonance
$\mathrm{BPH}$
Benign prostatic hyperplasia
TSP
Trimethylsilylpropionic acid- $\mathrm{d}_{4}$ sodium salt
CPMG
Carr-Purcell-Meiboom-Gill
FIDs
Free induction decays
1D
One-dimensional
2D
Two-dimensional
PQN
Probabilistic quotient normalization
PCA
Principal component analysis
OPLS-DA Orthogonal partial least squares discriminant analysis
VIP Variable importance in projection
BMI Body mass index
BCAA Branched-chain amino acids
LC-MS Liquid chromatography-mass spectrometry
GC-MS Gas chromatography-mass spectrometry
GNMT Glycine- $N$-methyltransferase
SARDH Sarcosine dehydrogenase 
DMGDH Dimethylglycine dehydrogenase

TCA Tricarboxylic acid

SDH Succinate dehydrogenase

FH Fumarate hydratase

\section{Introduction}

Prostate cancer $(\mathrm{PCa})$ is the most common cancer in men worldwide. The number of PCa cases is increasing, nowadays representing the sixth leading cause of cancer deaths in men (Zhang et al. 2014). Currently, the most frequently used tests for PCa screening include the determination of prostate specific antigen (PSA) serum levels and digital rectal examination (DRE) (Bunting 2002). The introduction of PSA testing revolutionised $\mathrm{PCa}$ screening and became widely adopted by the early 1990s. Since then, the European Randomized study of Screening for Prostate Cancer (ERSPC) has reported a small absolute survival benefit with PSA screening (Ilic et al. 2013; Heijnsdijk et al. 2015). However, PCa screening suffers from a number of limitations, due to the poor specificity of PSA test for detecting cancer and for differentiating indolent cancers from high risk ones.

The low specificity of serum PSA has translated into many unnecessary prostate biopsies and overtreatment of tumours with a low malignant potential, or with a low potential for morbidity or death if left untreated (Draisma et al. 2003; Zappa et al. 1998). It has been estimated that the overdiagnosis, and consequently the overtreatment, of PCa ranges between 30 and 84\%, depending on the studies (Etzioni et al. 2002; McGregor et al. 1998). Moreover, trans-rectal ultrasound (TRUS)-guided biopsy following histopathology-based Gleason score, the gold standard test providing histological confirmation (Gleason 1977), is also plagued by high false negative rates (Rabbani et al. 1998; Schoenfield et al. 2007). Early-stage PCa is generally not visible on ultrasound, thus meaning that many tumours are missed on initial biopsy and patients are required to undergo repeated prostate biopsies before definitive PCa detection.

Very few biomarkers are currently validated for use in $\mathrm{PCa}$ diagnosis. A recent FDA clinical-grade urine-based assay for the non-coding transcript $P C A 3$ (overexpressed in $>95 \%$ of $\mathrm{PCa}$ ) has demonstrated utility when combined with serum PSA for PCa detection (Loeb and Partin 2011). Another potential biomarker is the specific TMPRSS2 and ERG rearrangement at 21q22, which is $100 \%$ indicative of PCa (Barbieri et al. 2012). However, it is only present in approximately $50 \%$ of PCa cases. Hence, additional clinically robust biomarkers able to differentiate between indolent and aggressive PCa are urgently needed.

In this context, metabolomics could represent an alternative and very powerful approach for the understanding of the biological pathways and molecular mechanisms involved in the onset and progression of PCa. Metabolomics focuses on the characterisation of metabolic signatures in biofluids or tissues and is leading to advanced diagnostic and therapeutic procedures (Nicholson et al. 2005). Recent studies have shown the potential of metabolomic approaches in the PCa field (Kumar et al. 2015; Stabler et al. 2011; Struck-Lewicka et al. 2015; Zhang et al. 2013). However, so far, no comprehensive PCa studies have been performed on urine, the most accessible and least invasive biofluid, using Nuclear Magnetic Resonance ( ${ }^{1} \mathrm{H}-\mathrm{NMR}$ ) spectroscopy, a robust and reliable technological platform allowing the simultaneous measurement and quantification of metabolites with minimal sample handling (Duarte and Gil 2012).

To that end, in this study, a thorough analysis of the urinary metabolomic profile of PCa patients was compared with that corresponding to individuals diagnosed with benign prostatic hyperplasia (BPH), a prostatic condition that cannot be easily distinguished from $\mathrm{PCa}$ based on the current PSA screening (Roehrborn et al. 1999). Using a metabolomic approach based on ${ }^{1} \mathrm{H}-\mathrm{NMR}$, it was possible to identify a set of specific metabolites that could contribute to a better understanding of the pathophysiological processes involved in the onset and progression of this disease.

\section{Materials and methods}

\subsection{Patient selection}

Patient recruitment was carried out through the Department of Urology and the Biobank of the Instituto Valenciano de Oncología (Valencia, Spain), and measurement and analysis of the urinary metabolomic profiles were performed at the Centro de Investigación Príncipe Felipe (Valencia, Spain) and the Instituto de Investigación Sanitaria La Fe (Valencia, Spain). Urine samples were collected from $64 \mathrm{PCa}$ patients and 51 age-matched individuals. Patient recruitment and sampling procedures were performed in accordance with the Declaration of Helsinki and applicable local regulatory requirements and laws and after approval from the Ethics Committee of the Instituto Valenciano de Oncología. Written informed consent was obtained from each participant before being included in this study.

Clinical diagnosis of individuals was performed according to serum PSA, DRE, biopsy results and Gleason score. Biopsy was performed using at least 6 cores and classification of the individuals included in the study was carried out according to the EAU-ESTRO-SIOG Guidelines on Prostate Cancer (Mottet et al. 2016). The control group consisted of men with no proven PCa based on PSA levels, negative findings on DRE and no malignant findings in prostate tissue biopsies. Based on their clinical 
Table 1 Characteristics of the individuals included in the study

\begin{tabular}{lll}
\hline & $\begin{array}{l}\text { BPH group }(n=51) \\
\text { (median, range) }\end{array}$ & $\begin{array}{l}\text { PCa patients } \\
(n=64) \text { (median, } \\
\text { range) }\end{array}$ \\
\hline Age (years) & $62.1(41.4-74.5)$ & $66.2(50.0-86.3)$ \\
BMI $\left(\mathrm{kg} \mathrm{m}^{-2}\right)$ & $27(22.8-34)$ & $27.5(23-33)$ \\
Prostate volume $(\mathrm{ml})$ & $52(24-171)$ & $40.5(2-134)$ \\
PSA $(\mathrm{ng} / \mathrm{mL})$ & $4.86(1.02-11.29)$ & $5.11(0.85-71.41)$ \\
Number of cores & $12(10-19)$ & $12.5(6-54)$ \\
Positive cores & NA & $25 \%(0.05-100)$ \\
Tumor burden & NA & $3.71 \%(0.14-67.74)$ \\
Tumor gleason score & NA & $6(5-9)$ \\
\hline
\end{tabular}

$B P H$ benign prostatic hyperplasia, $P C a$ prostate cancer, $P S A$ prostatespecific antigen, $B M I$ body mass index, $N A$ not applicable

characteristics, all of them were diagnosed with $\mathrm{BPH}$. Clinical and demographics characteristics of the individuals included in the study are shown in Table 1.

\subsection{Sample preparation and ${ }^{1} \mathrm{H}-\mathrm{NMR}$ acquisition}

Urine samples were immediately frozen after collection and stored at $-80^{\circ} \mathrm{C}$. At the time of ${ }^{1} \mathrm{H}-\mathrm{NMR}$ analysis, urine samples were thawed on ice and centrifuged at $6000 \mathrm{rpm}$ for $5 \mathrm{~min}$ at room temperature. $60 \mu \mathrm{L}$ of $1.5 \mathrm{~mol} / \mathrm{L}$ potassium phosphate buffer ( $\mathrm{pH} 7.4$ ) containing $0.1 \%$ trimethylsilylpropionic acid-d $\mathrm{d}_{4}$ sodium salt (TSP) and $0.05 \% \mathrm{NaN}_{3}$ were added to $540 \mu \mathrm{L}$ of urine sample supernatant. After this, $500 \mu \mathrm{L}$ of the mixture were transferred to a $5-\mathrm{mm}$ NMR tube for analysis.

${ }^{1} \mathrm{H}-\mathrm{NMR}$ spectra were acquired using a Bruker Avance II $500 \mathrm{MHz}$ spectrometer. ${ }^{1} \mathrm{H}-\mathrm{NMR}$ experiments were acquired at $310 \mathrm{~K}$ for every sample. Carr-Purcell-Meiboom-Gill (CPMG) spin-echo pulse sequence (Meiboom and Gill 1958), which generates spectra edited by $\mathrm{T} 2$ relaxation times with reduced signals from high molecular weight species and giving improved resolution of low molecular weight metabolite resonances, was collected for each sample with a total of 16 accumulations and $72 \mathrm{~K}$ data points over a spectral width of $16 \mathrm{ppm}$. A 4-s relaxation delay was included between free induction decays (FIDs). The total spin-spin relaxation delay was $40 \mathrm{~ms}$. A onedimensional (1D) NOESY pulse sequence that generates an unedited spectrum with improved solvent peak suppression (Nicholson et al. 1995) was collected using the same parameters as the CPMG experiment, with a 4-s relaxation delay and $10 \mathrm{~ms}$ of mixing time. For both experiments, a water presaturation pulse of $25 \mathrm{~Hz}$ was applied throughout the relaxation delays to improve solvent suppression. In addition, two-dimensional (2D) J-resolved spectra, homonuclear $2 \mathrm{D}{ }^{1} \mathrm{H}-{ }^{1} \mathrm{H}$ total correlation spectroscopy and 2D ${ }^{1} \mathrm{H},{ }^{13} \mathrm{C}$ heteronuclear single quantum correlation were acquired for selected samples to facilitate the identification of biochmemical substances (Beckonert et al. 2007). All spectra were multiplied by a line-broadening factor of $1 \mathrm{~Hz}$ and Fourier transformed. Spectra were automatically phased and baseline corrected, and chemical shift internally referenced to the methyl group signal of TSP at $0.00 \mathrm{ppm}$ using TOPSPIN 3.0 (Bruker Biospin).

\subsection{Data modelling and statistical analysis}

The main steps of the data modelling and statistical analysis procedures followed in this study are shown in Fig. 1. 1D CPMG spectra were binned using Amix 3.9.7 (Bruker Biospin) into $0.001 \mathrm{ppm}$ wide rectangular buckets over the region $\delta 9.50-0.15 \mathrm{ppm}$. The water ( $\delta 5.09-4.55 \mathrm{ppm})$ and urea signal $(\delta 6.10-5.52 \mathrm{ppm})$ regions were excluded from the analysis to avoid interferences arising from differences in water suppression and variability from urea signal, respectively. Spectra were aligned using the "Speaq" $\mathrm{R}$ package, a hierarchical cluster-based peak alignment algorithm that minimizes chemical shift variations (Vu et al. 2011), and normalization of

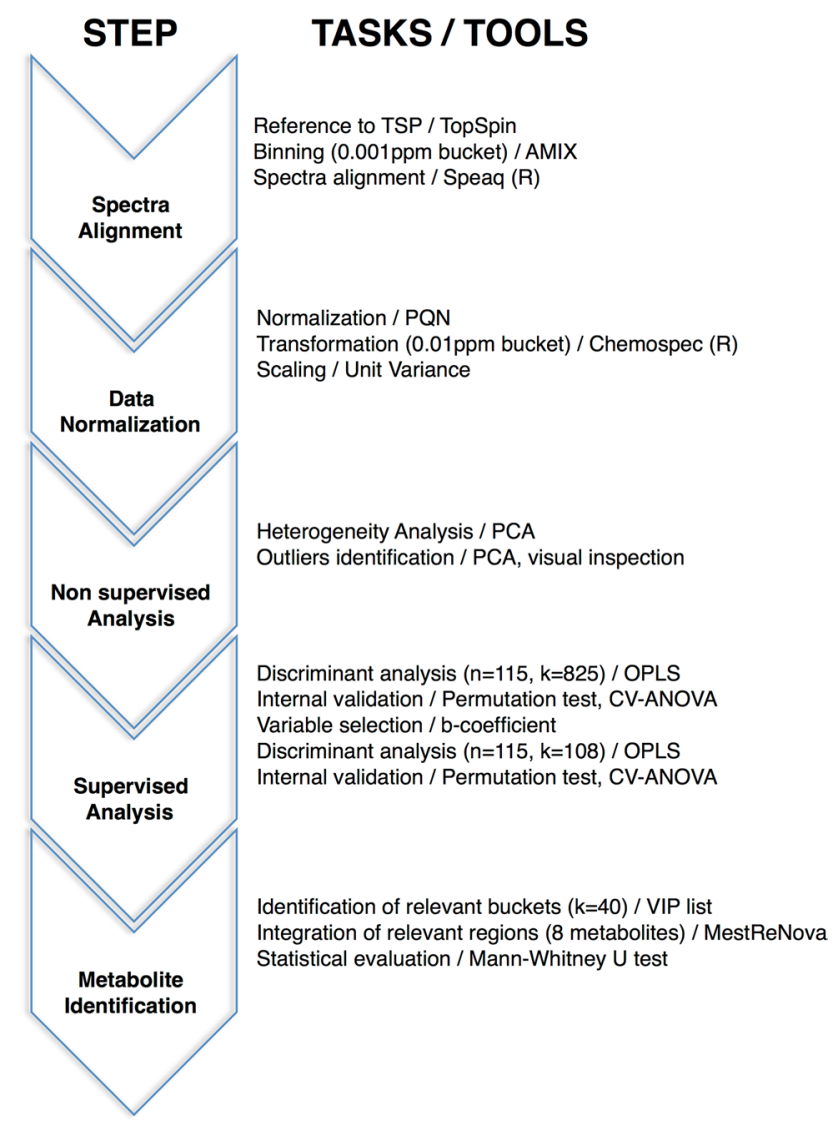

Fig. 1 General scheme of the data modeling and statistical analysis procedures with the main steps highlighted ( $n$ number of samples, $k$ number of variables) 
the aligned spectra was performed according to the probabilistic quotient normalization method (PQN) (Dieterle et al. 2006). Finally, the resulting bucket table was transformed into a data matrix containing $0.01 \mathrm{ppm}$ wide rectangular buckets using the "Chemospec" $\mathrm{R}$ package (Hanson 2014) to facilitate the statistical analysis.

Multivariate statistical analysis was carried out using SIMCA-P 12.0 (Umetrics AB). Before statistical analysis, data were scaled to unit variance by dividing each variable by $1 / \mathrm{SD}$, where SD represents the standard deviation value of each variable, so that all variables were given equal weight regardless of their absolute value. Principal component analysis (PCA), a nonsupervised statistical approach, was performed on normalized data for finding potential patterns, intrinsic clusters, and outliers. Orthogonal partial least squares discriminant analysis (OPLSDA) was applied to minimize the possible contribution of inter-group variability and to further improve separation between the groups of samples. The default method of sevenfold internal cross validation was applied, from which $Q^{2} Y$ (predictive ability parameter, estimated by cross-validation) and $R^{2} \mathrm{Y}$ (goodness of fit parameter) values were extracted. Those parameters, together with the corresponding permutation tests $(n=100)$, were used for the evaluation of the quality of the OPLS-DA models obtained. Variable selection was based on the regression coefficient (b-coefficient) method (Diaz et al. 2013), retaining only those variables with a quotient lb/ $b_{c v S E} \mid>1.0$, being $b_{c v S E}$ the standard error associated with the $b$-coefficients.

\subsection{Identification and quantification of relevant metabolites}

The identification of the variables responsible for the separation between groups of samples in the OPLS-DA models was performed according to the corresponding loading plots and the variable importance in projection (VIP) list of each model. Metabolites of interest were identified using Analysis of MIXtures (AMIX; Bruker) in combination with the Bruker NMR Metabolic Profiling Database BBIOREFCODE 2.0.0 database (Bruker Biospin, Rheinstetten, Germany), as well as other existing public databases and literature reports (Bouatra et al. 2013; Salek et al. 2007). Metabolites contributing to group discrimination in each model were integrated using MestReNova (Cobas and Sardina 2003) to enable comparison between sample groups. Statistical significance of the observed changes was assessed using the Mann-Whitney U test. A $p$ value lower than 0.05 (confidence level 95\%) was considered statistically significant.

\section{Results}

\subsection{Urinary metabolomic profile of PCa patients}

${ }^{1} \mathrm{H}-\mathrm{NMR}$ CPMG spectra were acquired for all urine samples included in the study. Good quality spectra, characterized by the presence of signals with varying degrees of overlapping, were obtained for most of the samples. Figure 2 displays a representative urine ${ }^{1} \mathrm{H}-\mathrm{NMR}$ spectrum from a PCa patient and the assignment of the most relevant metabolites identified in these samples. In general, spectra corresponding to this biofluid contain signals from a wide range of low-molecular-weight metabolites of diverse chemical classes (Bouatra et al. 2013), including organic acids, simple sugars and polysaccharides, amino acids, and low-molecular-weight proteins. In particular, urine spectra are dominated by urea, creatinine, trimethylamine- $N$-oxide, dimethylamine, hippuric acid, and citric acid resonances, among others (Fig. 2).

\subsection{Non-supervised analysis of the urinary metabolomic profiles}

Sample homogeneity within the groups of samples was based on the PCA analysis of the ${ }^{1} \mathrm{H}-\mathrm{NMR}$ CPMG urine spectra. Using this approach, it was possible to identify urine samples exhibiting metabolic profiles unusually different to the rest of the samples within their groups. Careful inspection of those samples revealed their spectra contained signals corresponding to several contaminants (e.g., manitol, ethanol, drugs, etc.), or exhibited bad quality due to acquisition problems. These samples were classified as outliers and excluded from the study.

PCA analysis was also used to evaluate the potential influence of different clinical variables on the metabolic profiles obtained for the urine samples of PCa patients and individuals diagnosed with $\mathrm{BPH}$. None of the variables assessed (i.e., age, PSA level, body mass index (BMI), Gleason score) had an impact in the clustering of the samples from both groups. Finally, a non-supervised analysis of the global data did not reveal any significant clustering of the samples based on the urine metabolomic profiles of the two sample groups in this study.

\subsection{Supervised analysis of the urinary metabolomic profiles}

To better examine potential differences between the groups of samples, an OPLS-DA model aiming to discriminate the urinary profiles from $\mathrm{PCa}$ patients and individuals diagnosed with BPH was built. This OPLS-DA model (Fig. 3) showed a reasonable fitting of the data $\left(\mathrm{R}^{2}=0.586\right)$, but it did not exhibit any predictive power $\left(\mathrm{Q}^{2}=-0.230\right)$. 

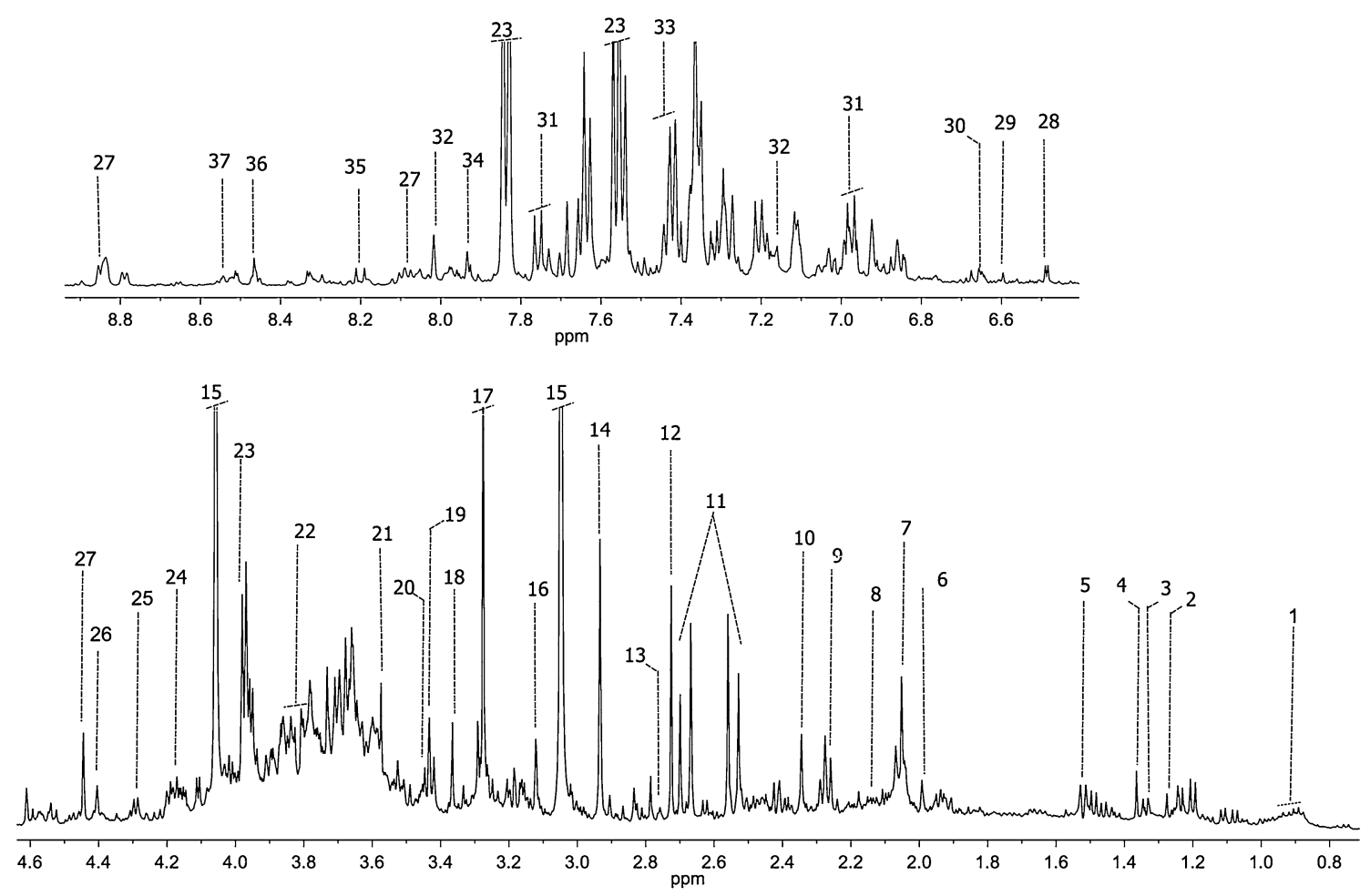

Fig. 2 Representative $500 \mathrm{MHz}{ }^{1} \mathrm{H}-\mathrm{NMR}$ spectrum and assignment of a urine sample from a PCa patient. Assigned metabolites: 1 branched-chain amino acids; 2 3-hydroxyisovalerate; 3 lactate; 4 2-hydroxyisobutyrate; 5 alanine; 6 acetate; $7 \mathrm{~N}$-acetyl groups; 8 glutamate; 9 2-hydroxy-glutarate; 10 pyruvate; 11 citrate; 12 dimethylamine; 13 sarcosine; 14 dimethylglycine; 15 creatinine; 16 cis-aco-

OPLS-DA model significance was assessed using a crossvalidated ANOVA ( $p \leq 0.01$ was considered significant) and a permutation test $(n=100)$. The results of this internal validation $\left(\mathrm{R}^{2}=0.600, \mathrm{Q}^{2}=-0.101 ; p\right.$ value $\left.>0.01\right)$ revealed overfitting of the data, most probably reflecting the elevated number of variables (823) over samples (115) used to build this model (Andersen and Bro 2010).

To overcome this limitation, a variable selection strategy, based on the regression coefficient method (b-coefficient) (Diaz et al. 2013), was followed to remove uninformative variables. The application of this variable selection method reduced the number of variables to 108 , and the OPLS-DA then provided a model with significant reduction in sample scores dispersion and improved predictive power $\left(\mathrm{Q}^{2}=0.416\right)$ (Fig. 4). The results of the internal validation of this new OPLS-DA model $\left(\mathrm{R}^{2}=0.358, \mathrm{Q}^{2}=-0.234\right.$; $p$ value $<0.01$ ) confirmed its robustness (Szymanska et al. 2012). The value of $R^{2}$ of this new model remained unchanged $\left(R^{2}=0.600\right)$ when compared with the original one, confirming that the discarded variables were not relevant for explaining the differences between the metabolomic profiles of $\mathrm{PCa}$ patients and individuals diagnosed with BPH. nitic acid; 17 trimethylamine- $N$-oxide; 18 methanol; 19 trans-aconitic acid; 20 taurine; 21 glycine; 22 serine; 23 hippurate; 24 pseudouridine; 25 threonine; 26 dihydroxyacetone; 27 trigonelline; $28 \mathrm{U1} ; 29$ fumarate; 30 2-furoylglycine; 31 4-hydroxybenzoate; 32 3-methylhistidine; 33 phenylalanine; 34 histidine; 35 hypoxanthine; 36 formate; 37 4-imidazole-acetate

\subsection{Metabolite identification and quantification}

Examination of the corresponding loading plot and VIP list of the new OPLS-DA model facilitated the identification of the most relevant variables that were contributing to the discrimination of the PCa patients and the individuals diagnosed with BPH. Following this strategy, a total of 40 out of the 108 variables were identified as relevant regions in the discrimination, and used to identify the spectral signals corresponding to the altered metabolites in pathological conditions. The metabolites corresponding to those regions were identified through a combination of their ${ }^{1} \mathrm{H}$ chemical shifts in the ${ }^{1} \mathrm{H}-\mathrm{NMR}$ CPMG spectra and the spin system patterns obtained from the 2D spectra acquired for representative samples of each group.

Further analysis of the data was carried out with the use of variable-size bucketing to assess if the metabolites associated with the relevant variables were also significant when comparing the two sample groups. This analysis revealed a total of 8 metabolites (Table 2) whose concentrations exhibited statistically significant differences when comparing the urinary metabolomic profiles of $\mathrm{PCa}$ patients and individuals diagnosed with BPH. Thus, it was 
Fig. 3 Multivariate modelling resulting from the analysis of urine ${ }^{1} \mathrm{H}$-NMR spectra before variable selection (823 variables). a OPLS-DA score plot for the comparison between $\mathrm{PCa}$ patients (red circle) vs. individuals diagnosed with BPH (blue circle); $\mathbf{b}$ internal validation of the corresponding OPLS-DA model by permutation analysis $(\mathrm{n}=100), \mathrm{R}^{2}$ (green diamond), $\mathrm{Q}^{2}$ (blue square)

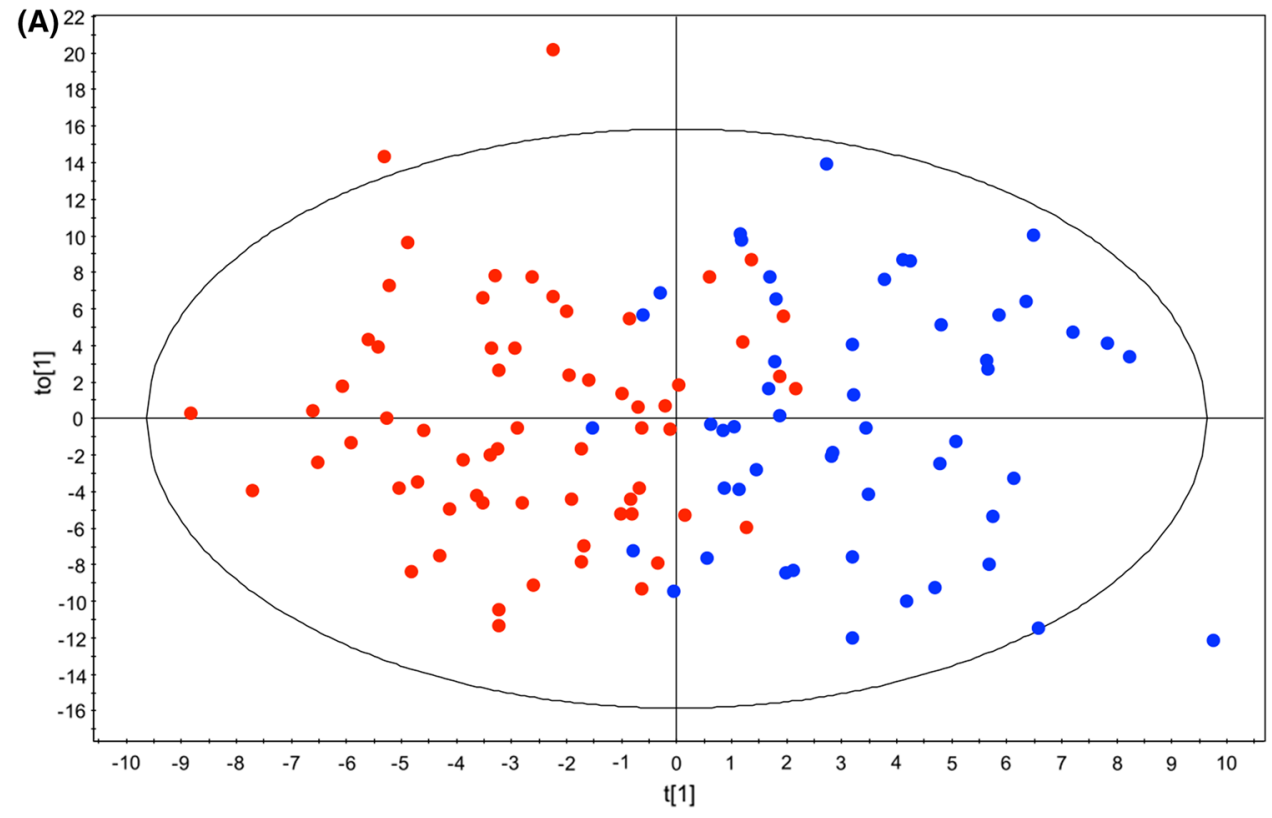

(B)

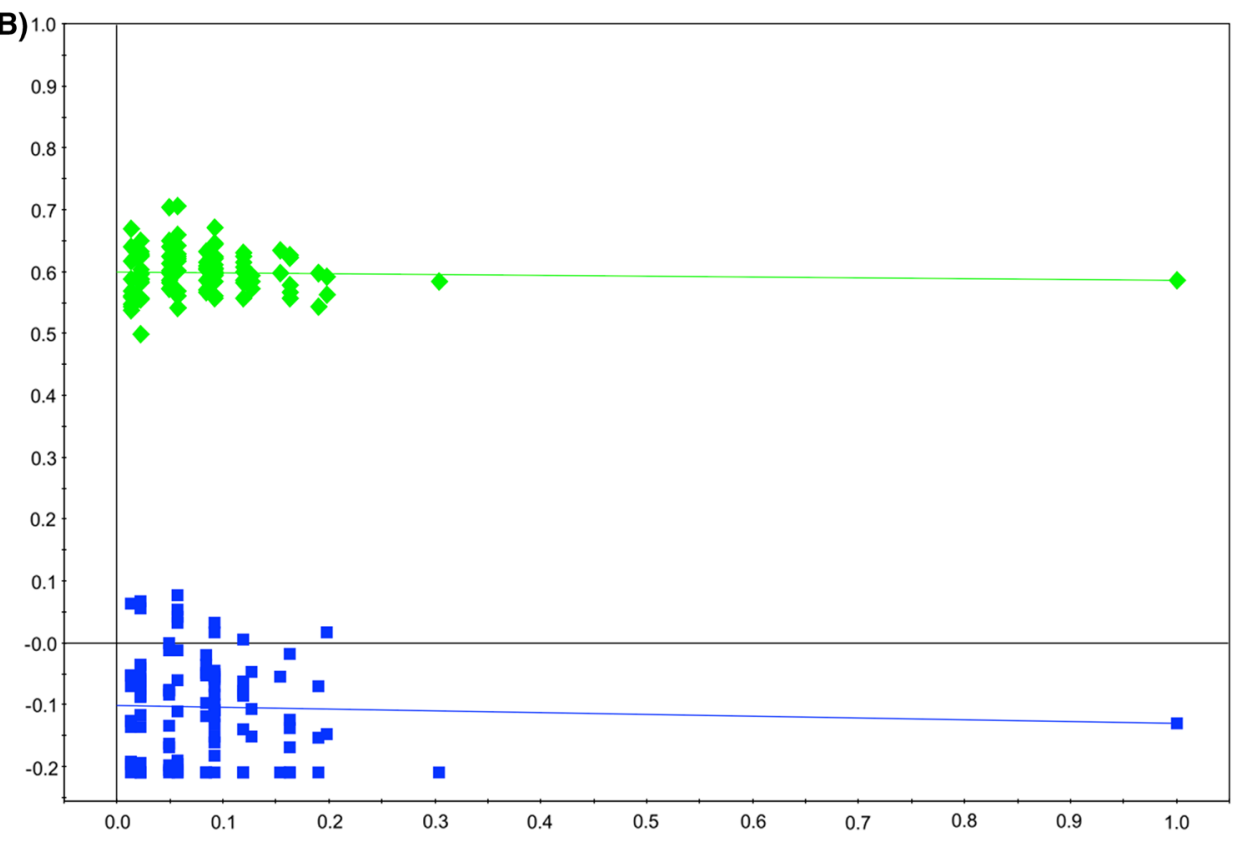

found that urine from PCa patients, compared with individuals diagnosed with BPH, was characterized by increased concentrations of branched-chain amino acids (BCAA), glutamate and pseudouridine, and decreased concentrations of glycine, dimethylglycine, fumarate, 4-imidazole-acetate, and one unknown metabolite (U1).

\section{Discussion}

Efforts to identify non-invasive PCa biomarkers that can stratify patients with high sensitivity and specificity for screening, diagnosis, prognosis, prediction and monitoring remain a fundamental goal in this area (Thapar and Titus 2014). In this context, our study represents the first comprehensive study focused on the characterisation and comparison of the specific urinary metabolomic profile of $\mathrm{PCa}$ patients with that of patients diagnosed with BPH using ${ }^{1}$ H-NMR. The only other report focused on the analysis of a relatively similar set of urine samples to that included in our study suggested that "fingerprints" (i.e., global profiles) based on the analysis of urinary NMR metabolomic profiles could be a suitable and promising method for $\mathrm{PCa}$ detection (Zaragoza et al. 2014). The in-depth analysis carried out in our study, based on non-invasive urinary metabolomic studies, reveals that the discrimination between PCa 
Fig. 4 Multivariate modelling resulting from the analysis of urine ${ }^{1} \mathrm{H}-\mathrm{NMR}$ spectra after variable selection (108 variables). a OPLS-DA score plot for the comparison between PCa patients (red circle) vs. individuals diagnosed with BPH (blue circle); $\mathbf{b}$ internal validation of the corresponding OPLS-DA model by permutation analysis $(\mathrm{n}=100), \mathrm{R}^{2}$ (green diamond), $\mathrm{Q}^{2}$ (blue square)

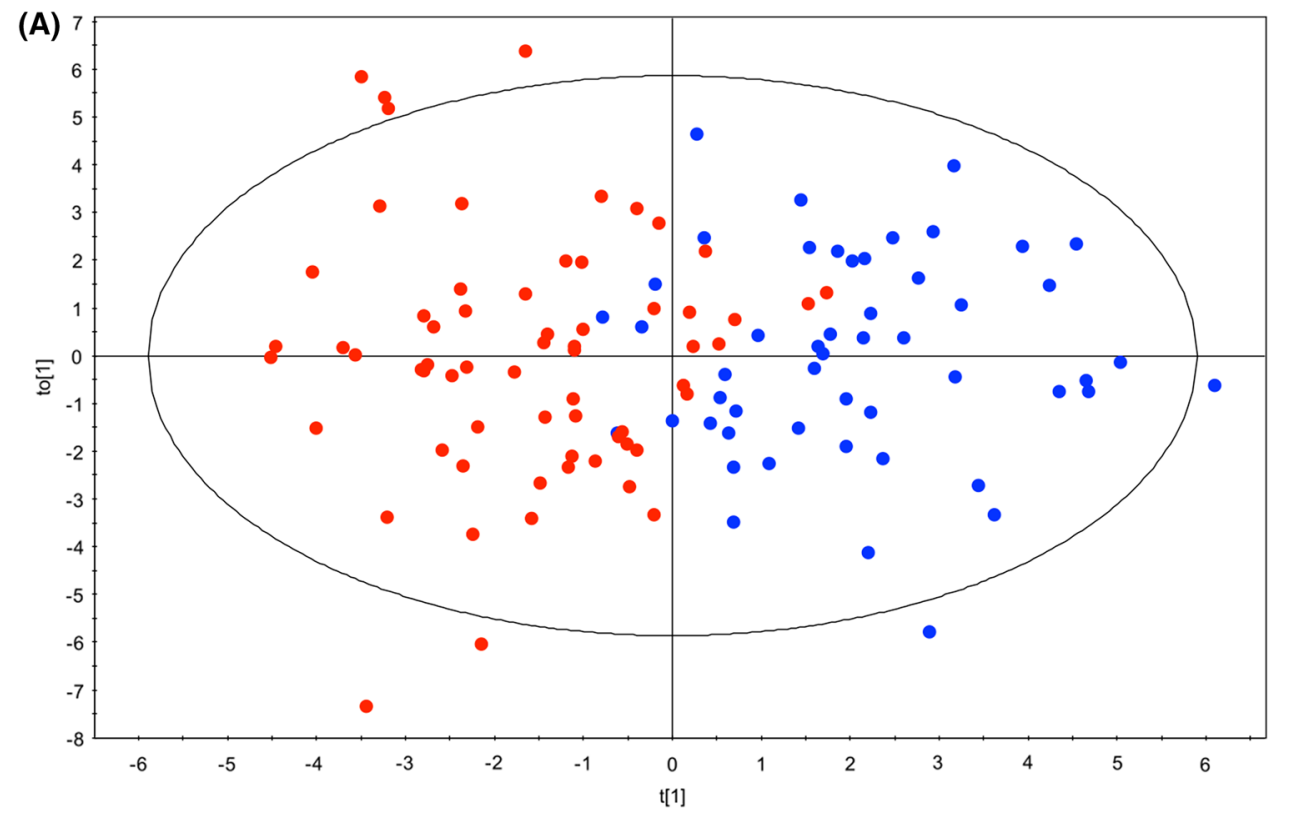

(B)

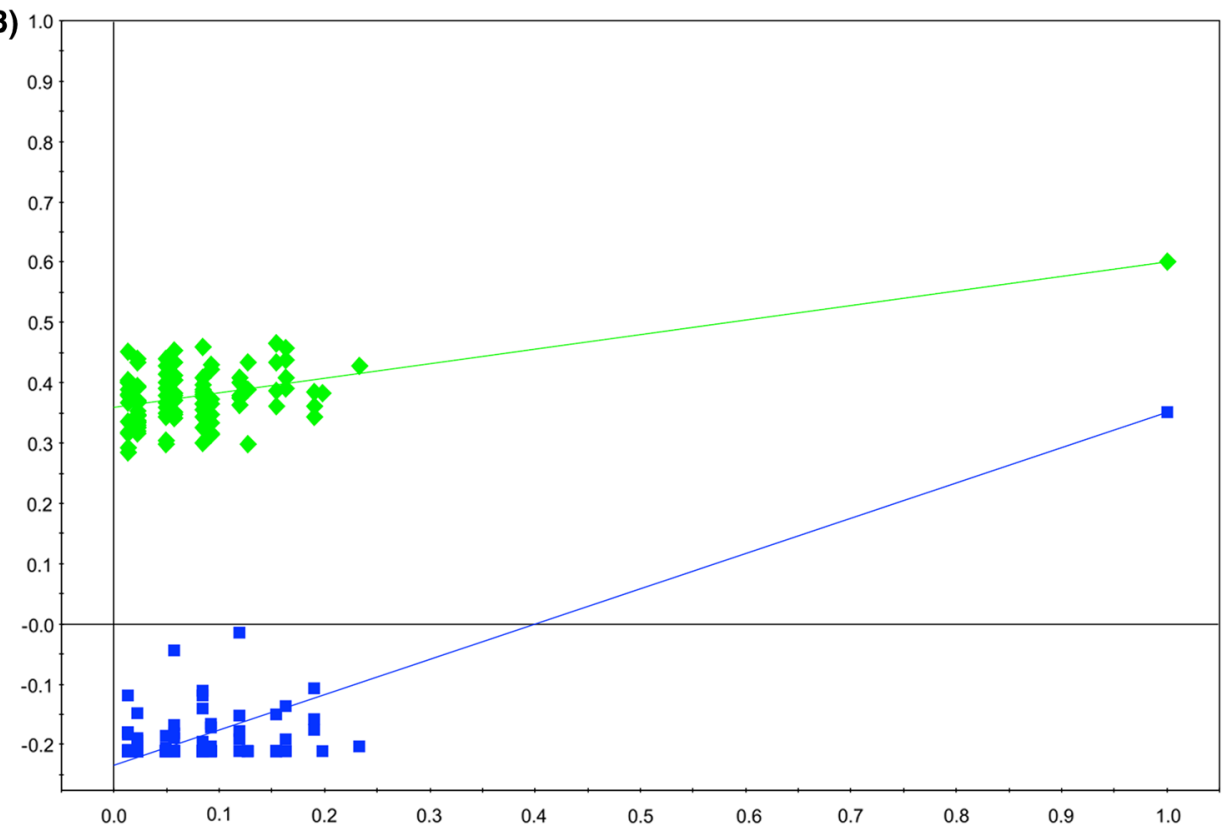

patients and individuals diagnosed with $\mathrm{BPH}$ actually relies on specific urinary metabolites, an information that could eventually contribute to the early diagnosis of PCa. Our results show that the urinary metabolomic profile of $\mathrm{PCa}$ patients, compared with individuals diagnosed with BPH, is characterised by statistically significant changes in the concentration of several metabolites. The analysis of those metabolic alterations reveals that $\mathrm{PCa}$ is associated with profound changes in energy metabolism.

Thus, in our study, we observed decreased levels of glycine and dimethylglycine when the urinary metabolomic profiles of PCa patients and individuals diagnosed with BPH were compared. This result is in agreement with recent studies performed in serum (Kumar et al. 2015) and urine (Struck-Lewicka et al. 2015) of PCa patients and healthy individuals. Kumar et al. (Kumar et al. 2015) found increased levels of sarcosine and decreased levels of glycine in serum samples of $\mathrm{PCa}$ patients compared with healthy individuals. Furthermore, Struck-Lewicka et al. (Struck-Lewicka et al. 2015) have reported decreased levels of glycine, in a study performed by liquid chromatography-mass spectrometry (LC-MS) and gas chromatography-mass spectrometry (GC-MS), when comparing the urinary metabolomic profiles of $\mathrm{PCa}$ patients and healthy individuals. 
Table 2 Mean intensities and variations for the statistically significant metabolites involved in the discrimination between individuals diagnosed with $\mathrm{BPH}$ and PCa patients

\begin{tabular}{lccccc}
\hline Metabolite & $\delta^{1} \mathrm{H}(\mathrm{ppm})^{\mathrm{a}}$ & $\begin{array}{l}\text { BPH group } \\
(\text { mean } \pm \text { s.e.m. })^{\mathrm{b}}\end{array}$ & $\begin{array}{l}\text { PCA patients } \\
(\text { mean } \pm \text { s.e.m. })^{\mathrm{b}}\end{array}$ & $p$ value & $\%$ variation \\
\hline BCAA & $0.930-0.842$ & $13.10 \pm 0.26$ & $14.33 \pm 0.37$ & $0.011^{*}$ & 9.37 \\
Glutamate & $2.115-2.081$ & $11.72 \pm 0.18$ & $12.42 \pm 0.21$ & $0.012^{*}$ & 5.98 \\
Dimethylglycine & $2.944-2.922$ & $20.56 \pm 0.91$ & $17.17 \pm 1.11$ & $0.034^{*}$ & -16.48 \\
Glycine & $3.582-3.567$ & $23.29 \pm 1.01$ & $20.49 \pm 0.94$ & $0.015^{*}$ & -12.00 \\
Pseudouridine & $4.309-4.277$ & $6.88 \pm 0.13$ & $7.68 \pm 0.41$ & $0.049^{*}$ & 11.65 \\
U1 & $6.496-6.478$ & $0.69 \pm 0.09$ & $0.45 \pm 0.04$ & $0.027^{*}$ & -34.50 \\
Fumarate & $6.551-6.498$ & $0.99 \pm 0.05$ & $0.87 \pm 0.03$ & $0.021^{*}$ & -12.54 \\
4-Imidazole-acetate & $8.567-8.517$ & $1.77 \pm 0.11$ & $1.37 \pm 0.62$ & $0.006^{*}$ & -22.52 \\
\hline
\end{tabular}

$B C A A$ branched-chain amino acids, ppm parts per million, s.e.m. standard error of mean. $P$ values calculated using the Mann-Whitney $\mathrm{U}$ test. $* P<0.05$

${ }^{a}$ Chemical shift range for integration

${ }^{\mathrm{b}}$ Spectral intensity in arbitrary units
Glycine is converted to sarcosine, an $N$-methyl derivative of glycine that has been previously linked to $\mathrm{PCa}$ (Sreekumar et al. 2009), by the enzyme glycine- $N$-methyltransferase (GNMT). Sarcosine levels are also regulated by sarcosine dehydrogenase (SARDH), the enzyme that converts sarcosine back to glycine, and dimethylglycine dehydrogenase (DMGDH) which generates sarcosine from dimethylglycine (Sreekumar et al. 2009). The involvement of sarcosine in PCa has been the subject of many studies (Khan et al. 2013; Miyake et al. 2012; Issaq 2011; Bianchi et al. 2011; Lucarelli et al. 2012; Sreekumar et al. 2009; Kumar et al. 2015; Jentzmik et al. 2010). However, its role as a potential biomarker of PCa remains controversial and unclear (Ploussard and de la Taille 2010). In our study, we found elevated levels of sarcosine in PCa patients, although this variation was not statistically significant. Taken together, our results would support the idea of an interconversion between glycine/dimethylglycine and sarcosine through the activation of both DMGH and GNMT, and the down-regulation of SARDH.

There are also other mechanisms that could contribute to a reduction in the levels of circulating glycine. Recent work on cancer metabolomics has shown that glycine uptake is associated with cancel cell proliferation through its involvement in one-carbon metabolism (Zhang et al. 2012). This pathway has been traditionally considered a "housekeeping" process, and encompasses a complex metabolic network based on the chemical reactions of folate compounds. Recent findings also suggests that hyperactivation of this pathway could potentially be a driver of oncogenesis and tumor maintenance (Locasale 2013). In this context, glycine metabolism has been reported to be involved in cell transformation and tumorigenesis. This process would be mediated by the activity of glycine dehydrogenase (decarboxylating) (GLDC) that links glycine cleavage with the charging of the folate cycle.
Furthermore, the rapid, dysregulated cell growth found in cancer cells, demands extra sources of energy to sustain proliferation (Zhang et al. 2012). Thus, in addition to pyruvate derived from glycolysis, fatty acids and particularly amino acids can supply substrates to the tricarboxylic acid (TCA) cycle to maintain mitochondrial production in cancer cells (Chen and Russo 2012).

One of the factors contributing to the availability of amino acids is a metabolic syndrome experienced by approximately $60 \%$ of PCa patients termed cachexia (Utech et al. 2012). This process involves a net increase in protein catabolism along with activation of proteolysis, and has a tremendous impact in the levels of BCAAs (O'Connell 2013). Under normal conditions, BCAA oxidation in skeletal muscle provides $6-7 \%$ of the energy needs, but under highly catabolic circumstances, such as cancer cachexia, the contribution can be as high as 20\% (Lam and Poon 2008). In these conditions, it would be expected an increase in circulating BCAAs, thus being in perfect agreement with our observation and other studies carried out in prostate tissue (Giskeødegård et al. 2013; McDunn et al. 2013) and serum samples (Giskeødegård et al. 2015) from PCa patients. It would also explain the results obtained in previous studies showing that the levels of BCAAs are significantly increased in certain neoplastic processes (e.g., gastric and esophageal cancers) (Fan et al. 2012; Zhang et al. 2013). Interestingly, BCAAs can be converted into acetylCoA and other organic molecules that enter the TCA cycle. The metabolic flexibility afforded by multiple inputs into the TCA cycle allows cancer cells to adequately respond to the fuels available in the changing microenvironment during the evolution of the tumor (Boroughs and DeBerardinis 2015).

Furthermore, the catabolism of BCAAs also provides an important source for the generation of amino acids, especially glutamine and alanine. Different cancer studies 
(Lasagna-Reeves et al. 2010; Gao et al. 2008; Zira et al. 2010) have shown alterations in glutamine levels that are presumably associated with increased metabolic activity derived from the conditions of hypoxia and hypermetabolism observed in the tumor environment (Eigenbrodt et al. 1998). Proliferating cancer cells take up glutamine and convert it to glutamate through a variet of deamidation and transamidation reactions, most notably the mitochondrial amidohydrolase glutaminase (Hensley et al. 2013). It leads to the production of ammonia and glutamate to balance the $\mathrm{pH}$ in tumor cells and could explain the increase of glutamate observed in the urine of PCa patients. This result is also in agreement with previous PCa studies performed in serum (Giskeødegård et al. 2015) and prostate tissue (McDunn et al. 2013). Glutamate is subsequently transformed into $\alpha$-ketoglutarate through a series of biochemical reactions termed glutaminolysis that contribute to replenish depleted intermediates of the TCA cycle (DeBerardinis et al. 2008).

Regarding amino acids metabolism, a significant decrease of 4-imidazole-acetate, a compound linked to histidine metabolism, was also observed in the urine of $\mathrm{PCa}$ patients. Interestingly, this metabolite was also identified in a previous study carrried out with serum samples collected up to 20 years prior to PCa diagnosis (Mondul et al. 2015). In this study, it was associated with both the overall risk of $\mathrm{PCa}$ (odds ratio 1.33) and aggressive $\mathrm{PCa}$ (odds ratio 1.40). Previous studies have also shown that histidine levels are increased in serum (Giskeødegård et al. 2015) and tissue (McDunn et al. 2013) samples from PCa patients, our finding perhaps reflecting a limited ability to process this amino acid by PCa cells. Alterations in histidine metabolism, as well as in BCAA (valine, leucine and isoleucine) metabolism, have also been observed in other cancers (e.g., ovarian cancer, breast cancer) (Ke et al. 2015; Schramm et al. 2010).

An increase in the urinary levels of pseudouridine, an isomer of the nucleoside uridine in which the uracil moiety is attached through a carbon-carbon bond, was found to be elevated in the urine metabolomic profile of $\mathrm{PCa}$ patients compared with individuals diagnosed with $\mathrm{BPH}$. Increased levels of uracil, or other uracil-containing metabolites (e.g., 2'-deoxyuridine) (Mondul et al. 2015), have been found in previous PCa studies (Jiang et al. 2010; McDunn et al. 2013; Spur et al. 2013; Sreekumar et al. 2009) suggesting an important role of the metabolism of this compound in this disease. Alterations in the levels of pseudouridine have also been observed in other pathological processes (Rasmuson and Bjork 1995; Vicente-Munoz et al. 2015; Masaki et al. 2006) and have been associated with disease activity, tumor burden, and clinical status (Tamura et al. 1987).

Finally, the analysis of the urinary metabolomic profiles of PCa patients and individuals diagnosed with BPH also revealed significant variations in the levels of fumarate, a key molecule in the TCA cycle. Within this cycle, the succinate dehydrogenase (SDH) complex converts succinate to fumarate, that is further down transformed to malate by the fumarate hydratase $(\mathrm{FH})$. Mutations in these enzymes have been previously linked to renal cell carcinomas, uterine and skin cancer (Tomlinson et al. 2002). Previous studies have also shown decreased levels of other TCA metabolites (isocitrate, aconitate and succinate) in the urine of PCa patients, all the data supporting a disruption in energy metabolism (Struck-Lewicka et al. 2015). Moreover, the decreased levels of fumarate in the urine of PCa patients, compared with individuals diagnosed with $\mathrm{BPH}$, positively correlates with previous studies showing an accumulation of this metabolite in PCa bone metastases (Thapar and Titus 2014) and prostate tissue (McDunn et al. 2013), a process that would lead to a reduction in the levels of circulating fumarate. Interestingly, succinate, another metabolite exhibiting decreased levels in the urine of PCA patients, also tends to accumulate in cancer cells. Both metabolites belong to a family of compounds termed oncometabolites that are known to accumulate in cancer cells and facilitate cancer progression (Yang et al. 2013). In particular, these two oncometabolites have been associated with the aberrant stabilization of HIF-1a (Semenza 2010), a key protein in cancer that is commonly overexpressed in $\mathrm{PCa}$ cells (Thomas and Kim 2008).

\section{Concluding remarks}

In summary, the present study reveals for the first time that the analysis of urinary metabolomic profiles provides a non-invasive tool for characterizing $\mathrm{PCa}$-associated biomarkers and for getting a better understanding of the metabolic alterations underlying this neoplastic process. Although further validation of the results, using an independent set of samples, will be necessary to increase the robustness of this analysis, our data support the idea that multivariate statistical analysis of ${ }^{1} \mathrm{H}-\mathrm{NMR}$ urinary metabolomic profiles obtained from PCa patients could be used for objectively discriminating individuals with $\mathrm{BPH}$ or $\mathrm{PCa}$.

Acknowledgements The authors thank all the staff members and the Biobank of the Instituto Valenciano de Oncología who contributed to sample collection and handling. Funding for the present study was provided by the Ministerio de Economía y Competitividad (SAF2014-53977-R), the Conselleria de Educación, Investigación, Cultura y Deporte (GVA, PROMETEO/2016/103) and a grant sponsored by Abbott from the Spanish Society of Urology (2012-2013). 


\section{Compliance with ethical standards}

Conflict of interest The authors declare that they have no conflict of interest.

Ethical approval All procedures performed in studies involving human participants were in accordance with the Declaration of Helsinki and applicable local regulatory requirements and laws and after approval from the Ethics Committee of the Instituto Valenciano de Oncología.

Ethical requirements The manuscript is in compliance with ethical requirement of the journal.

Informed consent Written informed consent was obtained from each participant before being included in this study.

Open Access This article is distributed under the terms of the Creative Commons Attribution 4.0 International License (http:// creativecommons.org/licenses/by/4.0/), which permits unrestricted use, distribution, and reproduction in any medium, provided you give appropriate credit to the original author(s) and the source, provide a link to the Creative Commons license, and indicate if changes were made.

\section{References}

Andersen, C. M., \& Bro, R. (2010). Variable selection in regressiona tutorial. Journal of Chemometrics, 24(11-12), 728-737.

Barbieri, C. E., Demichelis, F., \& Rubin, M. A. (2012). Molecular genetics of prostate cancer: Emerging appreciation of genetic complexity. Histopathology, 60(1), 187-198.

Beckonert, O., Keun, H. C., Ebbels, T. M., Bundy, J., Holmes, E., Lindon, J. C., et al. (2007). Metabolic profiling, metabolomic and metabonomic procedures for NMR spectroscopy of urine, plasma, serum and tissue extracts. Nature Protocols, 2(11), 2692-2703.

Bianchi, F., Dugheri, S., Musci, M., Bonacchi, A., Salvadori, E., Arcangeli, G., et al. (2011). Fully automated solid-phase microextraction-fast gas chromatography-mass spectrometry method using a new ionic liquid column for high-throughput analysis of sarcosine and $N$-ethylglycine in human urine and urinary sediments. Analytica Chimica Acta, 707(1-2), 197-203.

Boroughs, L. K., \& DeBerardinis, R. J. (2015). Metabolic pathways promoting cancer cell survival and growth. Nature Cell Biology, 17(4), 351-359.

Bouatra, S., Aziat, F., Mandal, R., Guo, A. C., Wilson, M. R., Knox, C., et al. (2013). The human urine metabolome. PLOS ONE, $8(9)$, e 73076.

Bunting, P. S. (2002). Screening for prostate cancer with prostate-specific antigen: Beware the biases. Clinica Chimica Acta; International Journal of Clinical Chemistry, 315(1-2), 71-97.

Chen, J. Q., \& Russo, J. (2012). Dysregulation of glucose transport, glycolysis, TCA cycle and glutaminolysis by oncogenes and tumor suppressors in cancer cells. Biochimica et Biophysica Acta, 1826(2), 370-384.

Cobas, J. C., \& Sardina, F. J. (2003). Nuclear magnetic resonance data processing. MestRe-C: A software package for desktop computers. Concepts in Magnetic Resonance Part A, 19A(2), 80-96.

DeBerardinis, R. J., Lum, J. J., Hatzivassiliou, G., \& Thompson, C. B. (2008). The biology of cancer: Metabolic reprogramming fuels cell growth and proliferation. Cell Metabolism, 7(1), 11-20.
Diaz, S. O., Barros, A. S., Goodfellow, B. J., Duarte, I. F., Galhano, E., Pita, C., et al. (2013). Second trimester maternal urine for the diagnosis of trisomy 21 and prediction of poor pregnancy outcomes. Journal of Proteome Research, 12(6), 2946-2957.

Dieterle, F., Ross, A., Schlotterbeck, G., \& Senn, H. (2006). Probabilistic quotient normalization as robust method to account for dilution of complex biological mixtures. Application in ${ }^{1} \mathrm{H}$ NMR metabonomics. Analytical Chemistry, 78(13), 4281-4290.

Draisma, G., Boer, R., Otto, S. J., van der Cruijsen, I. W., Damhuis, R. A., Schroder, F. H., et al. (2003). Lead times and overdetection due to prostate-specific antigen screening: Estimates from the European Randomized Study of Screening for Prostate Cancer. Journal of the National Cancer Institute, 95(12), $868-878$.

Duarte, I. F., \& Gil, A. M. (2012). Metabolic signatures of cancer unveiled by NMR spectroscopy of human biofluids. Progress in Nuclear Magnetic Resonance Spectroscopy, 62, 51-74.

Eigenbrodt, E., Kallinowski, F., Ott, M., Mazurek, S., \& Vaupel, P. (1998). Pyruvate kinase and the interaction of amino acid and carbohydrate metabolism in solid tumors. Anticancer Res, 18(5A), 3267-3274.

Etzioni, R., Penson, D. F., Legler, J. M., di Tommaso, D., Boer, R., Gann, P. H., et al. (2002). Overdiagnosis due to prostate-specific antigen screening: Lessons from U.S. prostate cancer incidence trends. Journal of the National Cancer Institute, 94(13), 981-990

Fan, J., Hong, J., Hu, J.-D., \& Chen, J.-L. (2012). Ion chromatography based urine amino acid profiling applied for diagnosis of gastric cancer. Gastroenterology Research and Practice, 2012, 474907.

Gao, H., Dong, B., Liu, X., Xuan, H., Huang, Y., \& Lin, D. (2008). Metabonomic profiling of renal cell carcinoma: High-resolution proton nuclear magnetic resonance spectroscopy of human serum with multivariate data analysis. Analytica Chimica Acta, 624(2), 269-277.

Giskeødegård, G. F., Bertilsson, H., Selnæs, K. M., Wright, A. J., Bathen, T. F., Viset, T., et al. (2013). Spermine and citrate as metabolic biomarkers for assessing prostate cancer aggressiveness. PLoS ONE, 8(4), e62375.

Giskeødegård, G. F., Hansen, A. F., Bertilsson, H., Gonzalez, S. V., Kristiansen, K. A., Bruheim, P., et al. (2015). Metabolic markers in blood can separate prostate cancer from benign prostatic hyperplasia. British Journal of Cancer, 113(12), 1712-1719.

Gleason, D. (1977). Histologic grading and clinical staging of prostaticcarcinoma. In M. Tannenbaum (Ed.), Urologic pathology: The prostate (pp. 171-198). Philadelphia, PA: Lea and Febiger.

Hanson, B. A. (2014). ChemoSpec: An R Package for Chemometric Analysis of Spectroscopic Data. Package Version 2.0-2.

Heijnsdijk, E. A., de Carvalho, T. M., Auvinen, A., Zappa, M., Nelen, V., Kwiatkowski, M., et al. (2015). Cost-effectiveness of prostate cancer screening: A simulation study based on ERSPC data. Journal of the National Cancer Institute, 107(1), 366.

Hensley, C. T., Wasti, A. T., \& DeBerardinis, R. J. (2013). Glutamine and cancer: Cell biology, physiology, and clinical opportunities. The Journal of Clinical Investigation, 123(9), 3678-3684.

Ilic, D., Neuberger, M. M., Djulbegovic, M., \& Dahm, P. (2013). Screening for prostate cancer. Cochrane Database of Systematic Reviews (Online), 1, CD004720.

Issaq, H. J., \& Veenstra, T. D. (2011). Is sarcosine a biomarker for prostate cancer? Journal of Separation Science, 34(24), 3619-3621.

Jentzmik, F., Stephan, C., Miller, K., Schrader, M., Erbersdobler, A., Kristiansen, G., et al. (2010). Sarcosine in urine after digital rectal examination fails as a marker in prostate cancer detection and identification of aggressive tumours. European Urology, 58(1), 12-18 (discussion 20-11). 
Jiang, Y., Cheng, X., Wang, C., \& Ma, Y. (2010). Quantitative determination of sarcosine and related compounds in urinary samples by liquid chromatography with tandem mass spectrometry. Analytical Chemistry, 82(21), 9022-9027.

Ke, C., Hou, Y., Zhang, H., Fan, L., Ge, T., Guo, B., et al. (2015). Large-scale profiling of metabolic dysregulation in ovarian cancer. International Journal of Cancer, 136(3), 516-526.

Khan, A. P., Rajendiran, T. M., Bushra, A., Asangani, I. A., Athanikar, J. N., Yocum, A. K., et al. (2013). The role of sarcosine metabolism in prostate cancer progression. Neoplasia, 15(5), 491-N413.

Kumar, D., Gupta, A., Mandhani, A., \& Sankhwar, S. N. (2015). Metabolomics-derived prostate cancer biomarkers: Fact or fiction? Journal of Proteome Research, 14(3), 1455-1464.

Lam, V. W., \& Poon, R. T. (2008). Role of branched-chain amino acids in management of cirrhosis and hepatocellular carcinoma. Hepatology Research, 38(Suppl 1), 107-115.

Lasagna-Reeves, C., Gonzalez-Romero, D., Barria, M. A., Olmedo, I., Clos, A., Sadagopa Ramanujam, V. M., et al. (2010). Bioaccumulation and toxicity of gold nanoparticles after repeated administration in mice. Biochemical and Biophysical Research Communications, 393(4), 649-655.

Locasale, J. W. (2013). Serine, glycine and one-carbon units: cancer metabolism in full circle. Nature Reviews Cancer, 13(8), 572-583.

Loeb, S., \& Partin, A. W. (2011). Review of the literature: PCA3 for prostate cancer risk assessment and prognostication. Reviews in Urology, 13(4), e191-e195.

Lucarelli, G., Fanelli, M., Larocca, A. M., Germinario, C. A., Rutigliano, M., Vavallo, A., et al. (2012). Serum sarcosine increases the accuracy of prostate cancer detection in patients with total serum PSA less than $4.0 \mathrm{ng} / \mathrm{ml}$. The Prostate, 72(15), 1611-1621.

Masaki, Y., Itoh, K., Sawaki, T., Karasawa, H., Kawanami, T., Fukushima, T., et al. (2006). Urinary pseudouridine in patients with lymphoma: Comparison with other clinical parameters. Clinica Chimica Acta, 371(1-2), 148-151.

McDunn, J. E., Li, Z., Adam, K. P., Neri, B. P., Wolfert, R. L., Milburn, M. V., et al. (2013). Metabolomic signatures of aggressive prostate cancer. The Prostate, 73(14), 1547-1560.

McGregor, M., Hanley, J. A., Boivin, J. F., \& McLean, R. G. (1998). Screening for prostate cancer: Estimating the magnitude of overdetection. Canadian Medical Association Journal, 159(11), $1368-1372$.

Meiboom, S., \& Gill, D. (1958). Modified spin-echo method for measuring nuclear relaxation times. The Review of Scientific Instruments, 29(8), 688-701.

Miyake M, G. G. E., Aguilar Palacios, D., \& Rosser, C. J. (2012). Sarcosine, a biomarker for prostate cancer: Ready for prime time? Biomarkers in Medicine, 6(4), 513-514.

Mondul, A. M., Moore, S. C., Weinstein, S. J., Karoly, E. D., Sampson, J. N., \& Albanes, D. (2015). Metabolomic analysis of prostate cancer risk in a prospective cohort: The alpha-tocolpherol, beta-carotene cancer prevention (ATBC) study. International Journal of Cancer, 137(9), 2124-2132.

Mottet, N., Bellmunt, J., Briers, E., Bolla, M., Cornford, P., De Santis, M., et al. (2016). EAU-ESTRO-SIOG guidelines on prostate cancer. European Association of Urology. doi:10.1016/j. eururo.2016.08.002.

Nicholson, J. K., Foxall, P. J., Spraul, M., Farrant, R. D., \& Lindon, J. C. (1995). $750 \mathrm{MHz}{ }^{1} \mathrm{H}$ and ${ }^{1} \mathrm{H}^{-13} \mathrm{C}$ NMR spectroscopy of human blood plasma. Analytical Chemistry, 67(5), 793-811.

Nicholson, J. K., Holmes, E., \& Wilson, I. D. (2005). Gut microorganisms, mammalian metabolism and personalized health care. Nature Reviews Microbiology, 3(5), 431-438.

O'Connell, T. M. (2013). The complex role of branched chain amino acids in diabetes and cancer. Metabolites, 3(4), 931-945.
Ploussard, G., \& de la Taille, A. (2010). Urine biomarkers in prostate cancer. Nature Reviews Urology, 7(2), 101-109.

Rabbani, F., Stroumbakis, N., Kava, B. R., Cookson, M. S., \& Fair, W. R. (1998). Incidence and clinical significance of false-negative sextant prostate biopsies. The Journal of Urology, 159(4), $1247-1250$.

Rasmuson, T., \& Bjork, G. R. (1995). Urinary excretion of pseudouridine and prognosis of patients with malignant lymphoma. Acta Oncologica, 34(1), 61-67.

Roehrborn, C. G., Boyle, P., Gould, A. L., \& Waldstreicher, J. (1999). Serum prostate-specific antigen as a predictor of prostate volume in men with benign prostatic hyperplasia. Urology, 53(3), 581-589.

Salek, R. M., Maguire, M. L., Bentley, E., Rubtsov, D. V., Hough, T., Cheeseman, M., et al. (2007). A metabolomic comparison of urinary changes in type 2 diabetes in mouse, rat, and human. Physiological Genomics, 29(2), 99-108.

Schoenfield, L., Jones, J. S., Zippe, C. D., Reuther, A. M., Klein, E., Zhou, M., et al. (2007). The incidence of high-grade prostatic intraepithelial neoplasia and atypical glands suspicious for carcinoma on first-time saturation needle biopsy, and the subsequent risk of cancer. BJU International, 99(4), 770-774.

Schramm, G., Surmann, E. M., Wiesberg, S., Oswald, M., Reinelt, G., Eils, R., et al. (2010). Analyzing the regulation of metabolic pathways in human breast cancer. BMC Medical Genomics, 3, 39 .

Semenza, G. L. (2010). HIF-1: Upstream and downstream of cancer metabolism. Current Opinion in Genetics and Development, 20(1), 51-56.

Spur, E. M., Decelle, E. A., \& Cheng, L. L. (2013). Metabolomic imaging of prostate cancer with magnetic resonance spectroscopy and mass spectrometry. European Journal of Nuclear Medicine and Molecular Imaging, 40(Suppl 1), 60-71.

Sreekumar, A., Poisson, L. M., Rajendiran, T. M., Khan, A. P., Cao, Q., Yu, J., et al. (2009). Metabolomic profiles delineate potential role for sarcosine in prostate cancer progression. Nature, 457(7231), 910-914.

Stabler, S., Koyama, T., Zhao, Z., Martinez-Ferrer, M., Allen, R. H., Luka, Z., et al. (2011). Serum methionine metabolites are risk factors for metastatic prostate cancer progression. PLOS ONE, 6(8), e22486.

Struck-Lewicka, W., Kordalewska, M., Bujak, R., Yumba Mpanga, A., Markuszewski, M., Jacyna, J., et al. (2015). Urine metabolic fingerprinting using LC-MS and GC-MS reveals metabolite changes in prostate cancer: A pilot study. Journal of Pharmaceutical and Biomedical Analysis, 111, 351-361.

Szymanska, E., Saccenti, E., Smilde, A. K., \& Westerhuis, J. A. (2012). Double-check: Validation of diagnostic statistics for PLS-DA models in metabolomics studies. Metabolomics, 8(Suppl 1), 3-16.

Tamura, S., Fujioka, H., Nakano, T., Hada, T., \& Higashino, K. (1987). Serum pseudouridine as a biochemical marker in small cell lung cancer. Cancer Research, 47(22), 6138-6141.

Thapar, R., \& Titus, M. A. (2014). Recent advances in metabolic profiling and imaging of prostate cancer. Current Metabolomics, 2(1), 53-69.

Thomas, R., \& Kim, M. H. (2008). HIF-1 alpha: A key survival factor for serum-deprived prostate cancer cells. The Prostate, 68(13), 1405-1415.

Tomlinson, I. P., Alam, N. A., Rowan, A. J., Barclay, E., Jaeger, E. E., Kelsell, D., et al. (2002). Germline mutations in FH predispose to dominantly inherited uterine fibroids, skin leiomyomata and papillary renal cell cancer. Nature Genetics, 30(4), 406-410.

Utech, A. E., Tadros, E. M., Hayes, T. G., \& Garcia, J. M. (2012). Predicting survival in cancer patients: The role of cachexia and 
hormonal, nutritional and inflammatory markers. Journal of Cachexia, Sarcopenia and Muscle, 3(4), 245-251.

Vicente-Munoz, S., Morcillo, I., Puchades-Carrasco, L., Paya, V., Pellicer, A., \& Pineda-Lucena, A. (2015). Nuclear magnetic resonance metabolomic profiling of urine provides a noninvasive alternative to the identification of biomarkers associated with endometriosis. Fertility and Sterility, 104(5), 1202-1209.

Vu, T. N., Valkenborg, D., Smets, K., Verwaest, K. A., Dommisse, R., Lemiere, F., et al. (2011). An integrated workflow for robust alignment and simplified quantitative analysis of NMR spectrometry data. BMC Bioinformatics, 12, 405.

Yang, M., Soga, T., \& Pollard, P. J. (2013). Oncometabolites: Linking altered metabolism with cancer. The Journal of Clinical Investigation, 123(9), 3652-3658.

Zappa, M., Ciatto, S., Bonardi, R., \& Mazzotta, A. (1998). Overdiagnosis of prostate carcinoma by screening: An estimate based on the results of the Florence Screening Pilot Study. Annals of Oncology: Official Journal of the European Society for Medical Oncology/ESMO, 9(12), 1297-1300.

Zaragoza, P., Ruiz-Cerda, J. L., Quintas, G., Gil, S., Costero, A. M., Leon, Z., et al. (2014). Towards the potential use of (1)H NMR spectroscopy in urine samples for prostate cancer detection. The Analyst, 139(16), 3875-3878.
Zhang, A., Yan, G., Han, Y., \& Wang, X. (2014). Metabolomics approaches and applications in prostate cancer research. Applied Biochemistry and Biotechnology, 174(1), 6-12.

Zhang, J., Bowers, J., Liu, L., Wei, S., Gowda, G. A., Hammoud, Z., et al. (2012). Esophageal cancer metabolite biomarkers detected by LC-MS and NMR methods. PLOS ONE, 7(1), e30181.

Zhang, T., Watson, D. G., Wang, L., Abbas, M., Murdoch, L., Bashford, L., et al. (2013). Application of holistic liquid chromatography-high resolution mass spectrometry based urinary metabolomics for prostate cancer detection and biomarker discovery. PLOS ONE, 8(6), e65880.

Zhang, W. C., Shyh-Chang, N., Yang, H., Rai, A., Umashankar, S., Ma, S., et al. (2012). Glycine decarboxylase activity drives nonsmall cell lung cancer tumor-initiating cells and tumorigenesis. Cell, 148(1-2), 259-272.

Zhang, X., Xu, L., Shen, J., Cao, B., Cheng, T., Zhao, T., et al. (2013). Metabolic signatures of esophageal cancer: NMR-based metabolomics and UHPLC-based focused metabolomics of blood serum. Biochimica et Biophysica Acta, 1832(8), 1207-1216.

Zira, A. N., Theocharis, S. E., Mitropoulos, D., Migdalis, V., \& Mikros, E. (2010). (1)H NMR metabonomic analysis in renal cell carcinoma: A possible diagnostic tool. Journal of Proteome Research, 9(8), 4038-4044. 